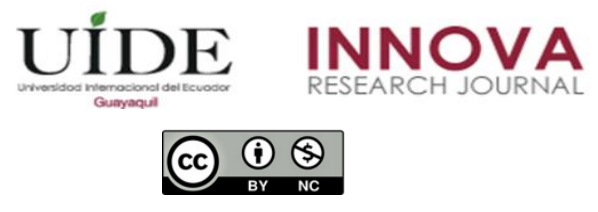

INNOVA Research Journal, ISSN 2477-9024

(Enero-Abril 2021). Vol. 6, No.1 pp. 162-176

DOI: https://doi.org/10.33890/innova.v6.n1.2021.1455

URL: http://revistas.uide.edu.ec/index.php/innova/index

Correo: innova@uide.edu.ec

\title{
Motivación laboral en una empresa de servicios de Lima, Perú
}

\section{Work motivation in a service company in Lima, Peru}

\author{
Fernando Alexis Nolazco Labajos \\ https://orcid.org/0000-0001-8910-222X \\ Elvis Iván Bustamante Cieza \\ (D) https://orcid.org/0000-0003-2093-724X \\ Rosa Ysabel Moreno Rodríguez \\ (D) https://orcid.org/0000-0002-8357-4514 \\ Irma Milagros Carhuancho Mendoza \\ D https://orcid.org/0000-0002-4060-5667 \\ Universidad Norbert Wiener, Perú
}

Autor para correspondencia: fernandonolazco@gmail.com; e.adm2015@ hotmail.com; rosa.moreno@uwiener.edu.pe; irma.carhuancho@uwiener.edu.pe

Fecha de recepción: 24 de junio de 2020 - Fecha de aceptación: 28 de octubre de 2020

\section{Resumen}

El talento humano es el recurso más importante y capaz de generar crecimiento en cualquier entidad. Por ello, compañías de todo tipo, tamaño, industria y ubicación direccionan sus esfuerzos en satisfacer sus necesidades creando espacios donde superen sus expectativas y logren sus satisfacciones. En ese contexto, en la compañía en estudio, no se evidenció una estructura de reconocimientos, prevalece una falta de capacitación, de oportunidades, de crecimiento y desarrollo. No existen estrategias para motivar al personal y/o espacios para el desarrollo de sus habilidades entre otras acciones. Por tal razón, el objetivo fue diagnosticar y determinar factores predominantes de la motivación laboral en los trabajadores de una empresa de servicios en Lima. El estudio se basó en un enfoque mixto, sintagma holístico, nivel comprensivo, método inductivodeductivo, la técnica fue la encuesta y entrevista apoyada de guía de entrevista, unidades informantes fueron treinta entre trabajadores y ex trabajadores y tres entrevistados. Los resultados evidencian desmotivación respecto a reconocimientos, bonos de incentivos, recompensas pero adicionales al salario y al aspecto de autonomía. En tanto manifestaron sentirse motivados con la cooperación entre áreas; la afiliación con los dueños, la marca y la interrelación con los colegas

generando un ambiente de confianza. Se consideran como factores predominantes la flexibilidad de horario, el feedback, integración, el fomento de investigación y apertura a nuevas formas de solución de problemas.

Palabras claves: motivación laboral; motivación intrínseca; motivación extrínseca; flexibilidad de horario; fomento de la investigación. 


\begin{abstract}
Human talent is the most important resource and capable of generating growth in any entity. Therefore, companies of all types, sizes, industries and locations direct their efforts to meet their needs by creating spaces where they exceed their expectations and achieve their satisfaction. In this context, in the company under study, there is no structure of recognition, there is a lack of training, opportunities, growth and development. There are no strategies to motivate the staff and/or spaces for the development of their skills among other actions. For this reason, the objective was to diagnose and determine predominant factors of work motivation in the workers of a service company in Lima. The study was based on a mixed approach, holistic syntagm, comprehensive level, inductive-deductive method, the technique was the survey and interview supported by interview guide, reporting units were thirty among workers and ex-workers and three interviewees. The results show a lack of motivation with respect to recognition, incentive bonuses, rewards but in addition to salary and the aspect of autonomy. The results show a lack of motivation with respect to recognition, incentive bonuses, rewards, but in addition to the salary and the aspect of autonomy. Flexible working hours, feedback, integration, promotion of research and openness to new forms of problem solving are considered to be predominant factors.
\end{abstract}

Keywords: work motivation; intrinsic motivation; extrinsic motivation; time flexibility; research promotion.

\title{
Introducción
}

La acción laboral desde sus orígenes a la actualidad ha sufrido considerables cambios. Consecuencia de ello, múltiples son los desafíos a los que se enfrentan los gerentes generales y gerentes de recursos humanos en departamentos de administración de la fuerza laboral. Por ejemplo, retos a escala de micro y macro entorno a la organización, aunada a ello, el de carácter profesional del trabajador. Más aún, si los trabajadores representan el recurso más importante en la esfera empresarial (William y Keith, 2008) y representan el medio que marca la diferencia en relación con otros recursos, siendo el único que a diferencia de las máquinas seguirá generando conocimiento según La Organización para la Cooperación y el Desarrollo Económicos y Ministerio de Trabajo y Promoción del Empleo (2016) y OCDE (2017). Por lo citado, lo predominante es hacer hincapié en el talento humano atendiendo las necesidades que repercuten en su motivación, dada pues su relevancia para el logro de objetivos tanto individuales como organizacionales. Razón por la cual, su reto es orientarse en prácticas que verdaderamente motiven a empleados de todas las edades como lo expresan (De Lange, Kooij y Van der Heijden, 2015; Kooij, De Lange, Jansen y Dikkers, 2013 citado por Kooij y Kanfer, 2019).

Particularmente, en estos tiempos dada la coyuntura nacional y mundial; donde las organizaciones están sistematizándose. Consecuencia de contexto actual y la dinámica del mercado global.

Como precedente al problema local, en lo nacional según Casana y Carhuancho (2019) en Perú, lo común es encontrar empresas que consideran lo económico como factor motivador, y es lógico. Sin embargo, ante un nuevo marco; los trabajadores no se sienten satisfechos; esto porque la motivación laboral va más allá de lo económico. Por lo que, es menester precisar que líderes de empresas deben prestar toda su asiduidad en la motivación del talento humano; y hacer que estos se sientan importantes, útiles, y puedan mejorar sus resultados al final de periodo, 
implementando nuevas técnicas de motivación laboral (Info Capital Humano, 2018; Rivera, 2019).

Aunque en menor proporción, diversas investigaciones han explorado cómo los factores externos e internos afectan o benefician la motivación de trabajadores. Por Ejemplo: en lo internacional, Rivas (2018) manifestó que los trabajadores perciben beneficios; pero no están acorde a la normativa vigente. Además, las personas no sienten que su esfuerzo sea valorado económicamente, tampoco su participación. Y, el ambiente de trabajo es desfavorable. Incluso, Sánchez, Sánchez, Cruz, y Sánchez (2014), concluyeron que, la satisfacción laboral se torna positiva o negativa a medida que las categorías: motivación, organización del trabajo, actividad, jornada laboral, supervisores, desarrollo profesional, vacaciones, promociones y permisos, se incrementa o disminuye. Es decir, si se incrementa la satisfacción laboral incrementa el rendimiento laboral y competitividad de la empresa.

En el ámbito nacional, Fernández (2018) fundamentó que la desmotivación responde a falta de reconocimiento, compensación inadecuada, falta de incentivos y recompensas. De manera tal que, el impacto en el desempeño, en la motivación intrínseca y en la motivación extrínseca del trabajador se ha visto influenciada. Resultando perjudicial (i) para trabajador y (ii) empresa. También, Chávez, Carhuancho, Nolazco, Venturo, y Miranda (2020) determinaron que los trabajadores efectúan sus actividades en espacios estrechos, repercutiendo en su concentración; la comunicación es limitada, brechas de desigualdad en relación con el salario. Sopesando a la capacitación como el principal factor de motivación laboral. Ergo, cualquier acción que limite su acceso, repercutirá directamente en su entusiasmo por trabajar, desempeño, actitudes y conductas de los trabajadores, lo cual se reflejará en la calidad de servicio.

En el ámbito local, tiene que trabajadores de la compañía de servicios, la misma que cuenta con más de siete años en la industria brindando servicios especializados; carecen de reconocimiento, capacitación, oportunidades de desarrollo profesional y planes de motivación. Además, se ha observado que los ambientes son compartidos. En consecuencia, la incomodidad de los trabajadores e insatisfacción se muestra a diario. Anexado a lo anterior, el deficiente control en la gestión económica, carencia de estrategias para motivar y falta de autonomía, incide su satisfacción. Sobre la base del conglomerado de problemas mencionados, la motivación laboral ha logrado representar el problema materia de investigación dado que la motivación laboral, la misma que se representa como un estado interno del trabajador para mantener y dirigir su conducta está conexo a todo lo postulado líneas arriba.

Respecto a fundamentos teóricos, el estudio se basa en la teoría de motivación-higiene fundamentada por Herzberg (1959) citado en Dalton, Hoyle, y Watts (2007) y Machuca (2009), ellos enfatizan que, la motivación del trabajador está condicionada en el ámbito laboral e influyen en la conducta laboral por dos grupos de factores, (a) los factores que se relacionan con el entorno donde los trabajadores desempeñan sus funciones, los llamó factores higiénicos; los cuales están fuera del control del trabajador. Por ejemplo: la compensación económica, beneficios, el clima de trabajo, entre otros. Y, el otro (b) relacionado con el aspecto intrínseco de la persona; es decir, todos los factores relacionados con el crecimiento, la libertad de acción, el progreso profesional, la responsabilidad, el reconocimiento y el logro. Agregando que si bien los 
factores de higiene no motivan, estos deben estar cubiertos para lograr la alta motivación del trabajador.

Otra línea de investigación, es la teoría de Daniel Pink (2009), el autor postula que se debe motivar al trabajador desde una perspectiva de la autonomía, basándose que la motivación intrínseca responde a la necesidad básica de sentir un grado de libertad para tomar decisiones dentro de la organización y dirigir su propia. Asimismo, la maestría, alega que es el resultado de las cosas repetitivas, la cual, permite al trabajador volverse cada vez mejor en lo que hace. Por último, el propósito, que hace referencia a que lineamientos estratégicos de la compañía deben en cierto nivel, estar en concordancia con los interese de los trabajadores. De ahí, la importancia de tener alineado la filosofía empresarial tanto para el trabajador como para la compañía (Pink, 2009; Universidad ORT Uruguay, 2016).

Concerniente al marco conceptual, la motivación laboral, comprende al conjunto de características tanto internas como externas positivas o negativas que influyen al momento de realizar una tarea o actividad; es decir, que repercute en el comportamiento del trabajador y, a su vez, influye en la satisfacción de una carencia o logro de objetivos personales, grupales o empresariales.

La motivación es un concepto muy complejo: "No solo implica características intrínsecas, sino que también está influenciada por la personalidad y las expectativas de cada individuo" (Singh, 2016, p. 198). Referente a la valoración, para Dalton et al. (2007), Ramírez, Abreu, y Badii (2008), Chávez et al. (2020) y Kodioglu y Yilmaz (2018); visualizan la definición desde el ámbito externo como interno y por la+ importancia a nivel empresarial, y como los objetivos muchas veces se logran por la voluntad del trabajador se requiere que estos tengan las herramientas y los factores que los motivan (Pizarro, Fredes, Inostroza, y Torreblanca, 2019). Desde el campo aplicativo, las conceptualizaciones dentro de la investigación aportaran para identificar, agrupar y valorar la motivación laboral de los trabajadores. Asimismo, conocer en primera instancia qué es lo que mueve al sujeto y cómo este afecta su desarrollo personal y aspiraciones.

Para Machuca (2009); Menges, Tussing, Wihler, y Grant (2017); Dalton et al. (2007); García (2005); De la Luz (2007); Cuesta (2015) y Chávez et al. (2020) la motivación laboral intrínseca es nativa del ser humano, lo que quiere decir que no es aprendido ni recibe refuerzo de un factor externo alguno, dado que la persona se auto refuerza. Por consiguiente, está bajo el control del individuo. Como indicadores se tiene al progreso individual, condiciones laborales, responsabilidad, autoestima, autonomía, motivo de trabajo, el logro y experiencia, entre otros.

En contraste, la motivación extrínseca hace referencia a los elementos externos, los cuales, que repercuten e influyen en la conducta del trabajador. Y, como indicadores se tienen a: los procedimientos y políticas de la compañía, seguridad de empleo, sueldo, bonificaciones, beneficios sociales, calidad de supervisión y relación de los supervisores, colegas y subordinados, condiciones físicas y ambientales de trabajo, forma de administración y decisión del trabajo, entre otro (Stringer, Didham, y Theivananthampillai, 2011; Machuca, 2009; Koontz, Weihrich, y Cannie, 2008; Chávez et al., 2020). 
En cuanto a la justificación teórica, la presente investigación se apoyará en la teoría de Daniel Pink y la teoría motivacional del Herzberg. En forma práctica, se justifica por su posterior aplicación para futuras investigaciones en la industria legal, así como también para hacer de conocimiento al empresariado, dirigentes, gerentes generales sobre los beneficios de alinear los objetivos empresariales con los del trabajador sobre la motivación laboral en la compañía, independientemente del tipo, tamaño, ubicación o actividad de esta. Metodológicamente se justifica sobre el diseño de investigación mixta, de sintagma holístico por el hecho que, se integrará o fusionará la información de carácter cuantitativa y cualitativa. Seguido de la interpretación y análisis de resultados consecuencia de la triangulación de la información. A fin de, presentar al mundo empresarial, en especial a los gerentes de los departamentos de recursos humanos y gerentes generales, aristas para reforzar vacíos existentes en la filosofía de motivación laboral que carezcan las compañías de servicios.

Para concluir esta parte del capítulo, la investigación tendrá como propósito general, diagnosticar el nivel motivación laboral para el íntegro de trabajadores que actualmente se desempeñan en la empresa de servicios. Siguiendo esa orden de aspiraciones, los objetivos específicos serán: (i) analizar el nivel de motivación laboral e (ii) identificar los factores motivacionales de mayor determinación.

\section{Figura 1}

Teorías relativas a la motivación en el trabajo

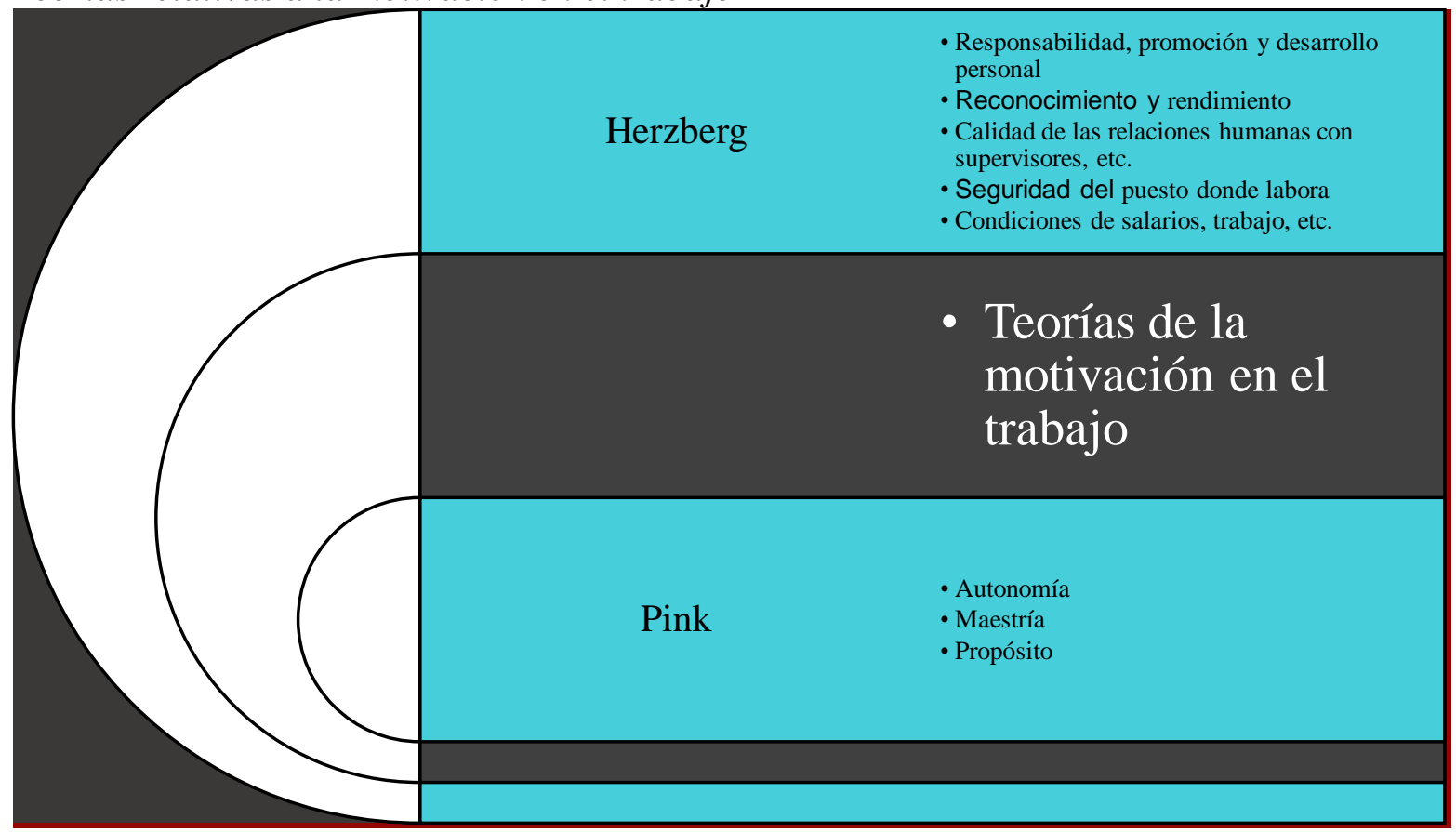

Elaboración propia 


\section{Método}

Se respalda en el enfoque mixto, sintagma holístico, esto porque se caracteriza según Carhuancho, Nolazco, Sicheri, Guerrero, y Casana, 2019; Hernández, Fernández, y Baptista (2014) por: la conceptualización desde la complejidad del problema y mezcla de métodos cualitativos y cuantitativos. Además, de nivel comprensivo, dado que faculta al investigador, el entendimiento, la interpretar y análisis de forma lógica y razonable de los resultados a fin de proponer aristas de solución a un escenario adverso donde la compañía se desenvuelve (Hurtado, 2004; Rodríguez y Peréz, 2017). La población-muestra para este estudio se tomó como referencia a 30 empleados y exempleados de la empresa de servicios; trabajando con el total de sujetos (Vara, 2012; Hernández y otros, citado por Carrasquero, 2014). Las unidades informantes lo conformaron un representante de cada departamento (contabilidad-administración y legal). En virtud de los instrumentos, la entrevista y se aplicó una encuesta online con 37 preguntas enfocadas en la motivación laboral. Además, se complementó la recopilación de información mediante la observación; esto, aplicando el principio de complementariedad como defiende (Carhuancho, 2020).

\section{Resultados}

En función de la interpretación de resultados cuantitativos, se encuestó a 30 trabajadores de los cuales 3 de ellos fueron fuente principal para la recopilación de testimonios sobre la motivación laboral en una empresa de servicios. Para agrupar a quienes proporcionaron la información, se clasificó en rango de edades, prevaleciendo los trabajadores de entre 18 y 30 años, alcanzando a un porcentaje del $63.3 \%$ del conglomerado de encuestados. En referencia a los hallazgos más relevantes sobre la subcategoría motivación laboral intrínseca; se determinó que los trabajadores se sienten motivados por el interés que tiene la empresa por su bienestar, los logros, éxitos y experiencias en la compañía, formar parte de una empresa de servicios y la necesidad de ascender y alcanzar metas profesionales y personales. No obstante, se sienten desmotivados por la falta de oportunidades para desarrollar sus capacidades y mejorar su situación laboral, pobre fomento de capacitaciones, limitada libertad para decidir y tomar decisiones de cómo hacer su trabajo, falta de integración en la toma de decisiones y finalmente falta de reconocimientos. Consecuencia de ello, el empresario debe adoptar acciones encaminadas por ejemplo a: fomentar un estilo de liderazgo democrático participativo y dar libertad de acción a su talento humano para que exploren sus habilidades y busquen nuevas formas de solución de problemas. Del mismo modo inculcar una cultura de inclusión en toma de decisiones y mayores reconocimientos, promover mayor oportunidades de integración e interconexión de conocimientos entre departamentos para fortalecer la motivación su motivación en el trabajo.

Respecto a la subcategoría motivación laboral extrínseca, se determinó que gran segmento de encuestados se mostró desmotivado con la claridad de sus funciones y responsabilidades, condiciones organizativas, distribución física de los equipos, bonos de incentivos y recompensas, pero adicionales al salario; las formas de despido y administración de la sociedad. Asimismo se determinó que el talento humano se muestra desmotivado con los procedimientos, normas, autonomía y acciones para motivar a los trabajadores, la libertad para 
desarrollar sus actividades y participar en el establecimiento de metas. En contraste, un importante segmento se sienten motivados por el sueldo que ganan por el trabajo realizado, las acciones en beneficio por sus familiares, la cooperación y coordinación entre departamentos, por la relación sólida con sus compañeros, supervisores y subordinados, el ambiente de confianza, la preocupación de los directivos por sus trabajadores. Finalmente, en lo que atañe a la afiliación, determinamos que los encuestados se sienten parcialmente motivados y altamente motivados con el vínculo sólido, sano y fuerte existente con los dueños, compañeros y marca de la compañía.

En lo que respecta al análisis de la motivación laboral intrínseca, en los testimonios más relevantes; los entrevistados enfatizaron que consecuencia del estilo de dirección en muchas ocasiones se hace lo que dice el jefe o dueño de la compañía; resultado perjudicial para su entusiasmo y libertad de acción. Más aún, para el cliente. Enfatizando que la libertad de acción debe primar en todos los niveles organizativos, puesto que les origina un sentido de pertenencia e integración. Con referencia a los reconocimientos, incentivos y recompensas los entrevistados infieren no sentirse satisfechos; valorando que el impulso de alguna forma de reconocimiento por parte de la compañía, resulta motivador. No obstante, este reconocimiento no necesariamente puede ser económico, puesto que los reconocimientos pueden resultar perjudiciales y costosos para la compañía e incrementa la competitividad e individualismo.

Por otra parte, consideran que la organización y planeación es fundamental, puesta que al ser una empresa, las áreas trabajan sistemáticamente; por lo que una mala planeación y organización en sus actividades puede desencadenar entornos tensos. Aunado a las situaciones antes descritas, se determinó al feedback, las recompensas y ascensos como factores motivadores. Por otra parte, es necesario buscar formas más dinámicas de trabajo y evitar lo rutinario. Valorando de forma significativa las capacitaciones y oportunidades de desarrollo como factores que infieren en su motivación. Al mismo tiempo afirman, que la empresa debe ser un centro donde se fomente la investigación, la capacitación constante y el aprendizaje continuo.

En contraste, lo que atañe al análisis de la subcategoría motivación laboral extrínseca; en relación con la forma de administración y decisión del trabajo, consideran que al estar bajo las riendas de la familia les genera un sentir de insatisfacción. Agregando que las decisiones en la empresa deberían ser más horizontales. Enfatizan además, falta de incentivos, beneficios o bonificaciones; rescatando como factor motivador, la flexibilidad de horario. En otro contexto, la cooperación entre áreas es excelente, gratificante, buena y entusiasta. Por lo que atañe a la afiliación, los vínculos con los compañeros, la marca y dueños de la compañía es lo más sólido. Agregando que debe primar la interconexión, el fomento de la investigación y capacitaciones para el desarrollo de habilidades. Además, su deseo es encontrar en la empresa un lugar donde se fomente la participación e integración, la apertura en la búsqueda de nuevas formas de resolución de labores y problemas. 


\section{Figura 2}

Análisis de la motivación laboral (nube de palabras)

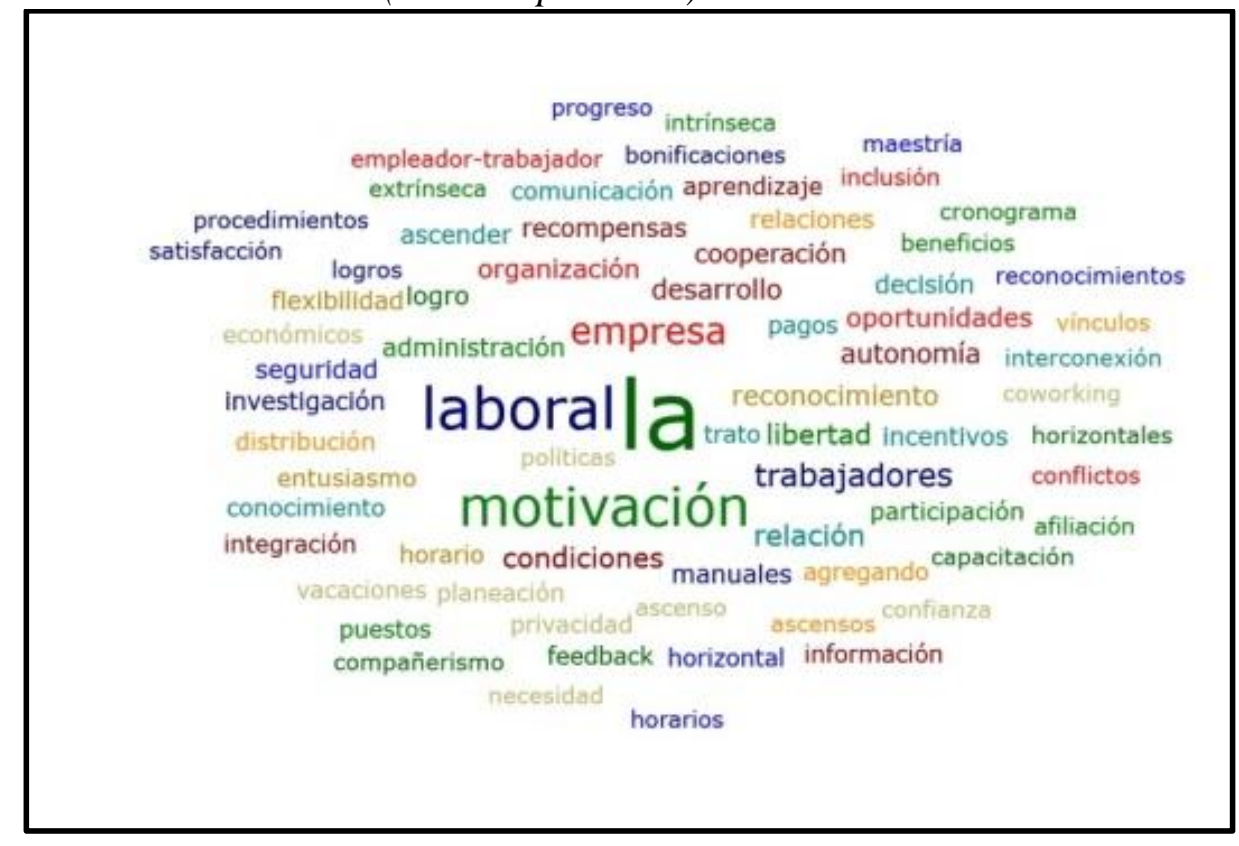

En la figura se puede observar con claridad la conjugación en la palabra motivación laboral y todas las palabras claves que están conexas a ella. Por ejemplo, flexibilidad, horario, autonomía, libertad, oportunidades, administración, entusiasmo, integración, feedback, necesidad, recompensas, relación: empleador - trabajador, comunicación, entre otras.

Desde un diagnóstico final; la motivación laboral de los trabajadores de una empresa de servicios; se ha respaldado de dos subcategorías (i) motivación laboral intrínseca (MLI) y (ii) extrínseca (MLE). Reforzando a cada subcategoría con indicadores que a continuación analizaremos los más resaltantes; desde la perspectiva de la triangulación de la información brindada por los agentes informantes: Partiendo de la subcategoría MLI, se tiene como indicador al reconocimiento; se determinó que los trabajadores sienten insatisfacción, que existe una carencia de reconocimientos, incentivos y recompensas por el trabajo realizado; esto se evidencia, puesto que los encuestados se sienten desmotivados. 


\section{Figura 3}

\section{Análisis de la categoría motivación laboral y su subcategoría motivación intrínseca}

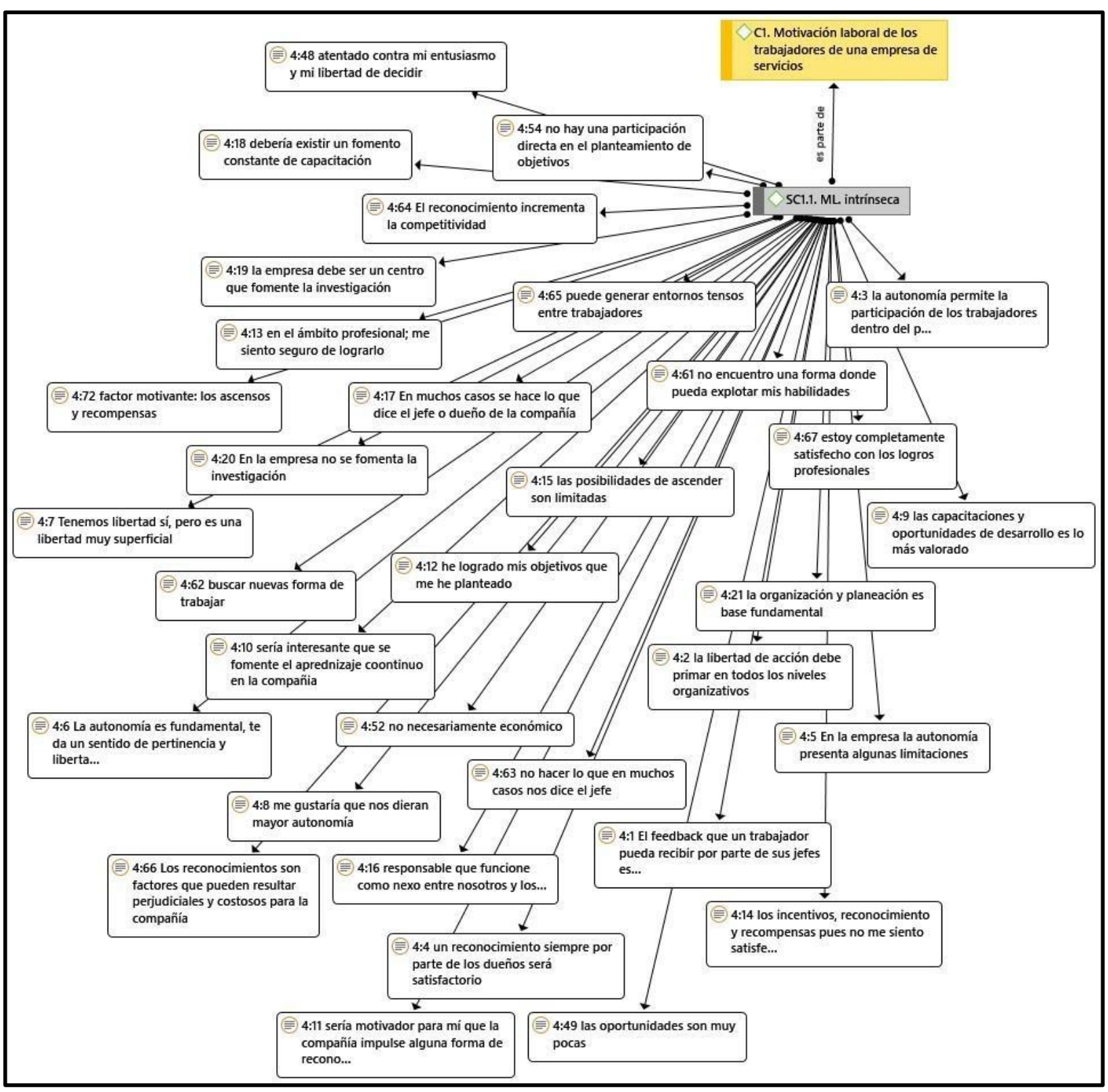

Esta obra se comparte bajo la licencia Creative Common Atribución-No Comercial 4.0 International (CC BY-NC 4.0) 
Ante ello, uno de los entrevistados; refirió que un reconocimiento por parte de los dueños de la compañía resulta satisfactorio. No obstante, este reconocimiento no necesariamente puede ser económico; esto porque incrementa la competitividad e individualismo y pueden resultar perjudicial y costoso para la compañía. Con referencia al indicador autonomía, un preocupante segmento se mostró desmotivado. Se ratifica este resultado, puesto que en los testimonios se determinó que en muchos casos se hace lo que dice el jefe o dueño de la compañía. Dicho acto, limita esa facultad para realizar sus actividades. Aunado a ello, sienten no tener libertad para decidir cómo hacer sus tareas. Consecuencia de ello, un entrevistado manifestó que en muchos casos ha resultado perjudicial para la compañía y sobre todo para el cliente. La motivación laboral de los trabajadores se ve promovida por factores tanto internos como externos; por ello se requiere el fomento de la participación de los trabajadores en las decisiones de la compañía y se les dé autonomía para realizar su trabajo (Bernal y Sierra, 2013).

Anexado a los indicadores anteriores tenemos al progreso, manifiestan no encontrar en la empresa una forma donde puedan explorar sus habilidades; y más aún, si las capacitaciones y oportunidades de desarrollo son lo que según los entrevistados, más valoran; determinándose con respecto a las oportunidades para desarrollar sus capacidades que en su mayoría se muestran desmotivados Para Herzberg (1950) citado por Machuca (2009), para proporcionar motivación laboral continua (MLC) determina que se debe enriquecer las tareas ofreciendo al trabajador condiciones de satisfacción y desafío profesional, para que mediante este mecanismo el trabajador pueda continuar con su crecimiento individual. Atendiendo a estas carencias, los empleados manifiestan que la empresa debe ser un centro donde encuentren espacios para el desarrollo de sus habilidades, recomendando la creación de contextos donde simulen realizar informes orales de casos judiciales. 


\section{Figura 4}

\section{Análisis de la categoría motivación laboral y su subcategoría extrínseca}

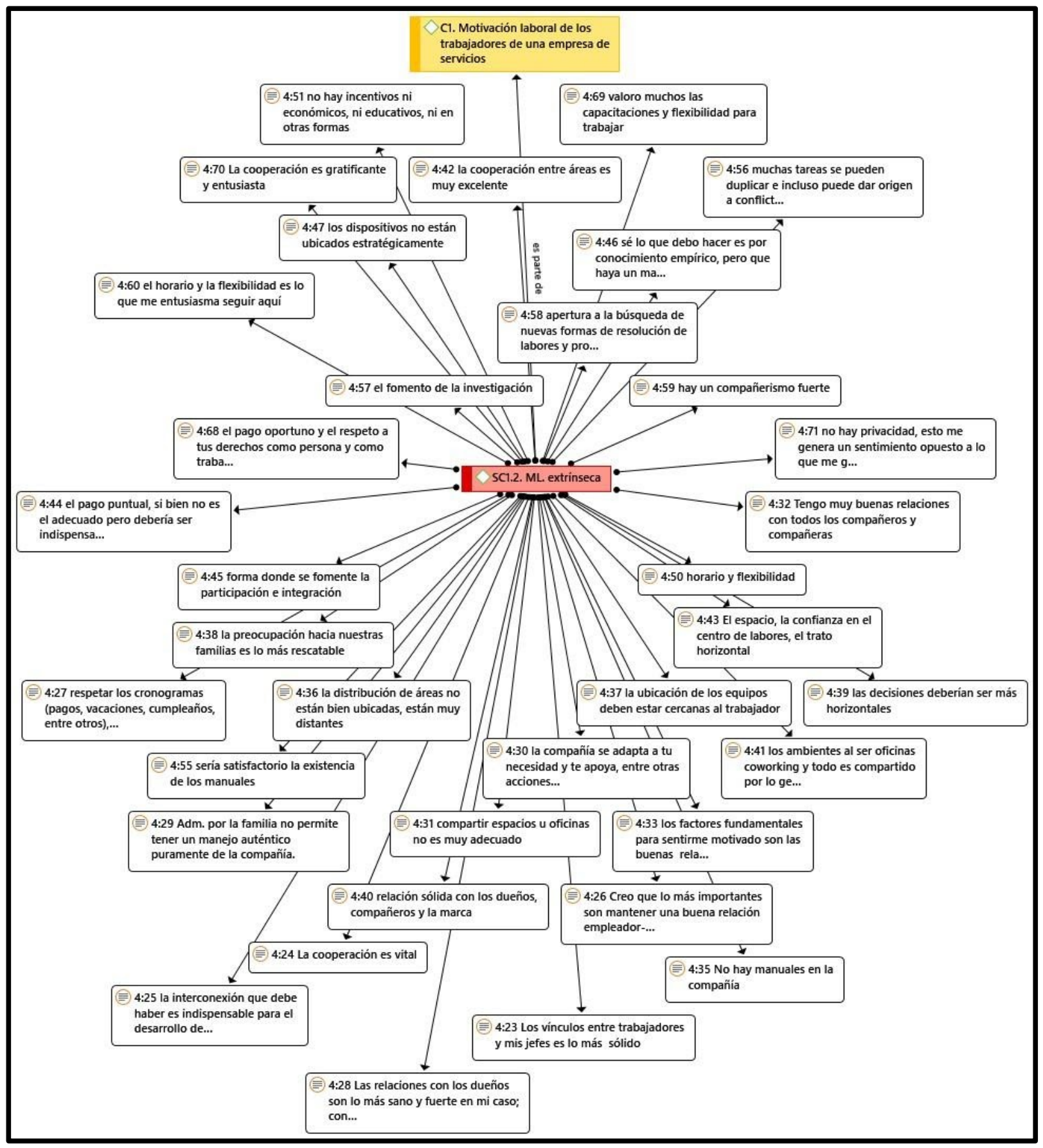

Por lo que se refiere a la motivación laboral extrínseca, la misma que se apoya en indicadores como: sueldo, bonificaciones y beneficios sociales, un sólido segmento atañe que se 
encuentra motivado en mención al sueldo que perciben por el trabajo realizado. No obstante, están desmotivados por los bonos de incentivos y recompensas adicionalmente al salario, esto se refuerza dado que un entrevistado refirió que no hay incentivos; ni recompensas en ninguna modalidad. También se evidenció respecto a la afiliación, que un sólido segmento indica sentirse motivados con el vínculo existente con los compañeros de trabajo, con la marca y con los dueños; reforzamos estas apreciaciones, puesto que los entrevistados refieren que ese lazo existente entre ellos es lo más fuerte y sano.

Por lo último, según McClelland citado por Bernal y Sierra (2013), la afiliación como necesidad que motiva a los seres humanos, se manifiesta en el anhelo de los trabajadores por conservar y crear amistades evitando conflictos; por ello prefieren situaciones de colaboración sobre las individuales o competitivo. Resulta lógico esta idea, puesto que los trabajadores han manifestado la existencia de una excelente y entusiasta cooperación entre departamentos de trabajo. Asimismo, manifiestan evitar la competitividad e individualismo. Es preciso indicar también que este enfoque, está conexo al indicador calidad de supervisión, relación con los supervisores, colegas y subordinados, donde determinamos que cerca de la totalidad de trabajadores se sienten motivados, consolidándose más aún con los testimonios donde manifestaron que uno de los factores por lo que se sienten motivados son las buenas relaciones laborales, la existencia de un compañerismo fuerte y la cooperación entre áreas es excelente, gratificante, buena y entusiasta. Aunado a ello, manifiestan una mayor oferta de oportunidades de integración e interconexión; puesto que se encuentra parcialmente desmotivado con la participación en decisiones en la empresa.

En relación con el indicador forma de administración y decisión del trabajo: los entrevistados se muestran parcialmente desmotivado, manifestando que al estar administrada por la familia le genera insatisfacción, no permite tener un manejo autentico, puro de la compañía; se tiende a combinar las gestiones empresa-familia. Agregando que las políticas deben ir en un sentido de integración adoptando mayor libertad de acción para que los trabajadores exploren sus habilidades. Finalmente, refieren que la retroalimentación que pueda recibir es un factor motivador; la apertura en la búsqueda de nuevas formas de resolución de labores y el pago puntual. Asimismo, el fomento de la investigación, las capacitaciones e incluso consideran que la empresa debe ser un centro donde se fomente la investigación y participación. Concluyendo que las recompensas y ascensos son factores motivadores.

Para Ibáñez (2011) en su libro “Gestión del Talento Humano en la Empresa”, el proporcionar retroalimentación (retroalimentación) al trabajador de su rendimiento, de su área y de la misma forma de la empresa, es una regla de motivación por el jefe. Por ello, es importante mantener al trabajador informado acerca de su desempeño. Independientemente de que el resultado sea negativo o positivo, el autor considera que hasta de la peor de las situaciones se puede sacar algo positivo.

\section{Discusión}

Los resultados obtenidos en el presente estudio titulado "Motivación laboral en los trabajadores en una empresa de servicios, Lima 2020”, armonizan con los precedentes y 
enfoques previamente citados; en los diferentes análisis e interpretaciones de la motivación laboral, la misma que se representa como un estado interno que mantiene y dirige el accionar del trabajador. Asimismo, se muestra influenciada por factores internos y externos al propio empleado Stringer et al. (2007). En esa misma línea, un factor trascendental es la autonomía, en los testimonios se evidenció que en muchos casos los trabajadores hacen lo que dice el jefe. Consecuentemente, no sienten tener libertad para decidir cómo hacer su trabajo, lo cual, está en contra de lo que plantea en su teoría Herzberg citado por Dalton et at. (2007) y el enfoque de motivación de Pink citado por Cirino (2015); donde refieren que la motivación laboral extrínseca responde a la necesidad de sentir un grado de libertad de acción.

Por otra parte, y según los resultados, las capacitaciones son lo que, entre otras, más valoran, evidenciándose desmotivación respecto a este elemento, el cual conjuga con el estudio de Chávez et al. (2020) donde manifiesta entre otros que cualquier acción que limite el acceso a capacitaciones repercutirá directamente en su entusiasmo por trabajar, reflejado en la calidad del servicio. Consecuentemente, alterará el desempeño, las actitudes y conductas del trabajador. También prevalece el reconocimiento, evidenciándose una falta de reconocimientos adicionales al salario, este resultado armoniza con lo expuesto por Fernández (2018) quien fundamentó que la desmotivación responde a una falta de reconocimiento, incentivos y recompensas; y también lo establecido por Casana y Carhuancho (2019), aduciendo que, en un nuevo entorno, la motivación va más allá de lo económico.

También, la población en estudio se mostró desmotivados por una falta de bonificaciones y beneficios sociales; si bien son factores que están fuera de control del trabajar, estos necesitan estar cubiertos si se quiere lograr que el talento humano esté altamente motivado (Herzberg citado por Dalton et al., 2007). Otro factor trascendental es el vínculo con los compañeros, la marca y dueños de la compañía, evidenciándose que existe un lazo sólido y fuerte. Asimismo, la cooperación entre ellos y departamentos es entusiasta y excelente y les gustaría que se preserve, el cual armoniza por lo planteado por McClelland citado por Bernal \& Sierra (2013), refiriendo a la afiliación como una necesidad que responde al anhelo del trabajador por conservar y crear amistades evitando conflictos, por ello prefieren situaciones de colaboración.

\section{Conclusiones}

En la empresa materia de investigación, se determinó que el nivel de motivación laboral de los trabajadores difiere de unos a otros según los enunciados en la encuesta. Por ejemplo: con relación a las recompensas, incentivos y reconocimiento se sienten parcial y altamente desmotivados, de igual forma en relación con los bonos de incentivos adicionales al salario, con las oportunidades de desarrollo profesional, participación en toma de decisiones, fomento de capacitaciones y condiciones organizativas. Aunado a ello, se mostraron parcial y altamente desmotivados con la autonomía y libertad de acción. En contraste, están parcial y altamente motivados con sus logros, la cooperación y coordinación entre departamentos, con el ambiente de confianza, con la preocupación de los directivos por su bienestar, con el vínculo existente entre ellos y los dueños de la compañía, los trabajadores y la marca. Esta situación, obliga a los directivos a sofisticar la gestión del talento, no como una moda sino como una necesidad que obliga a estructurar procesos y políticas centradas en la motivación laboral para seguir 
alcanzando metas, objetivos y servir mejor al cliente. En esa misma línea, se determinó como factores determinantes entro otros al, flexibilidad de horario, la retroalimentación, las capacitaciones, apertura a nuevas formas de solución de labores y problemas, la participación e integración, el fomento de la investigación en la empresa, oportunidades de desarrollo profesional. Se recomienda hacer hincapié en el enriquecimiento de tareas y en estructurar reconocimientos adicionales al salario.

\section{Referencias Bibliográficas}

Bernal, A. (2010). Metodología para la investigación: administración, economía, humanidades y ciencias sociales (Tercera ed.). Colombia: Pearson Educación.

Carhuancho Mendoza, I. M. (17 de febrero de 2020). Tarea de Texto argumentativo. Obtenido de Archivo de video: Recuperado de https://www.youtube.com/watch?v=djKbD473UCA

Carhuancho, I., Nolazco, F., Sicheri, L., Gerrero, M. \& Casana, K. (agosto de 2019). Metodología para la investigación holística. Obtenido de https://repositorio.uide.edu.ec/bitstream/37000/3893/3/Metodolog\%c3\%ada\%20para\%20 la\%20investigaci\%c3\%b3n\%20hol\%c3\%adstica.pdf

Carrasquero, A. (2014). Holistic research and instructional development in unsdestanding written discourse in students average eduction. (J. Féliz Blaco, Ed.) Revista de Investigación , 81(38), 68-88.

Casana, K., \& Carhuancho, I. (2019). Análisis de la gestión del talento humano en una institución pública, en Perú. Investigação Qualitativa em Ciências Sociais/Investigación Cualitativa en Ciencias Sociales, 3, 120-125.

Chávez, F., Carhuancho, I., Nolazco, F., Venturo , C., \& Miranda, B. (2020). Motivation of public service in a municipality in Peru. International Journal of Scientific \& Technology Research, 211-219.

Cuesta, A. (2015). Gestión del talento humano y el conocimiento. Colombia: Macro.

Dalton, M., Hoyle, D., \& Watts, M. (2007). Relaciones Humanas. México: Thomson.

Daniel, P. (2009). Drive: The surprising truth about what motivates Us. España: Planeta.

De la Luz, A. (2007). Recursos humanos en la empresa. Puerto Rico: Puertoriqueñas.

Fernández, A. (2018). Estrategias para mejorar la motivación laboral en un instituto técnico superior. (Tesis de licenciatura). Universidad Norbert Wiener, Lima. Obtenido de http://repositorio.uwiener.edu.pe/xmlui/bitstream/handle/123456789/2483/TESIS\%20Fer nandez\%20Augusto.pdf? sequence $=1 \&$ isAllowed $=\mathrm{y}$

García , J. (2005). El comportamiento humano en las organizaciones. Perú: Universidad del Pacífico.

Hernández, R., Fernández, C. \& Baptista, P. (2014). Metodología de la investigación (Sexta ed.). México: McGraw-Hill.

Hurtado, J. (2004). Cómo formular objetivos de investigación. Bogotá, Colombia : Cooperativa Editorial Magisterio.

Ibáñez, M. (2011). Gestión del talento humano en la empresa. Perú: San Marcos.

Info Capital Humano (2018). Perú es el tercer país con mayor rotación de personal en Latam.

Kodioglu, H., \& Yilmaz, P. (2018). Investigation of the work motivation levels of primary school teachers. Journal of Education and Training Studies, 184-196. 
Kooij, D., \& Kanfer, R. (2019). Lifespan perspectives on work motivation. Researchegate, 475793.

Koontz, H., Weihrich, H. \& Cannie, M. (2008). Administración. Una perspectiva global y empresarial. México: McGrawHill.

Machuca, A. (2009). Las organizaciones del siglo XXI. Apuntes de Administración General. Perú: San Marco.

Menges , J., Tussing, D., Wihler, A., \& Grant, A. (2017). When job performance is all relative: How family motivation energizes effort and compensates for intrinsec motivation. Academy of Management Journal, 60(2), 695-719.

OCDE (2017). Perspectivas económicas de América Latina 2017. Juventud, competencias y emprendimiento, 2017.

OCDE \& MTPE (2016). Estrategia de competencias de la OCDE. Reporte Diagnostico: Perú, 2016.

Pizarro, J., Fredes, D., Inostroza , C., \& Torreblanca, E. (2019). Motivación, satisfacción laboral y estado de flow en los trabajos de salud. Revista Venezolana de Gerencia, 24(87).

Ramírez, R., Abreu, J. \& Badii, M. (2008). La motivación laboral, factor fundamental para el logro de objetivos organizacionales. International Journal of Good Conscience, 143-185.

Rivas, A. (2018). La motivación laboral como detonante para el mejor desempeño en las pequeñas empresas de la ciudad de Puebla. (Tesis de maestría). Benemérita Universidad Autónoma de Puebla, México.

Rodríguez, A. \& Peréz, A. (2017). Métodos científicos de indagación y de construcción del conocimiento. EAN, 82, 179-200.

Sánchez, M., Sánchez, P., Cruz, M. \& Sánchez, F. (2014). Características organizacionales de la satisfacción laboral en España. RAE-Revista de Administración de Empresas, 54(5), 537547.

Singh, R. (2016). The impact of intrinsec and extrinsic motivators on employees engagement in information organizations. Journal of Education for Library and Information Science , 197-206.

Stringer, C., Didham, J. \& Theivananthampillai, P. (2011). Does pay satisfaction affect motivation and job satisfaction?. Qualitative Research in Accounting \& Management,.

Universidad ORT Uruguay (25 de febrero de 2016). Claves para la motivación en el trabajo: cómo inspirar a las personas. Obtenido de Archivo de video: Recuperado de https://www.youtube.com/watch?v=RSq1SrY5ON0

Vara, A. (2012). Desde la idea hasta la sustentación: Siete pasos para una tesis exitosa. Un Método efectivo para las ciencias empresariales. (Tercera). Lima: Universidad San Martín de Porres.

William, B. \& Keith, D. (2008). Administración de Recursos Humanos. El Capital Humano de las Empresas.

Obtenido

de https://cucjonline.com/biblioteca/files/original/c2f2989d851e80e2cc6aa0ebf3a54cb0.pdf 